\title{
Diffusion-weighted imaging as a follow-up modality for evaluation of major salivary gland function in nasopharyngeal carcinoma patients: a preliminary study
}

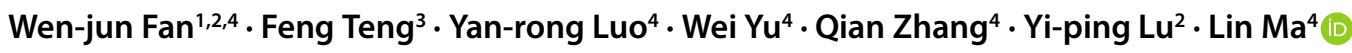 \\ Received: 12 October 2019 / Accepted: 7 January 2020 / Published online: 5 February 2020 \\ (c) The Author(s) 2020
}

\begin{abstract}
Purpose To investigate the value of diffusion-weighted imaging (DWI) in assessing dynamic changes of major salivary gland function during follow-up post radiotherapy (RT) in nasopharyngeal carcinoma (NPC) patients.

Materials and methods 31 consecutive patients with pathologically confirmed NPC scheduled for RT underwent six routine follow-up MRI examinations including DWI sequence prior to (pre-RT) and 1, 3, 6, 9, and 12 months post RT. Mean apparent diffusion coefficient (ADC) values of bilateral parotid glands (PGs) and submandibular glands (SMGs) were measured. Objective measurement of salivary flow rate (SFR) under unstimulated (uSFR) and stimulated conditions (sSFR) as well as subjective xerostomia assessment according to a patient-rated questionnaire were conducted before each MRI. Variance analysis was used to evaluate dynamic changes of ADC, SFR and xerostomia questionnaire summary scores (XQ-sum) at different timepoints and the correlation between ADC and XQ-sum. Pearson's correlation test was used to evaluate the correlations between pre- and post-RT changes of ADC ( $\triangle$ ADC) and SFR $(\triangle \mathrm{SFR})$ or mean RT dose.

Results At each timepoint, ADCs of PGs were significantly lower than of SMGs, uSFR was significantly lower than sSFR. For both PGs and SMGs, $\mathrm{ADC}_{\text {post-RT }}$ were all higher than $\mathrm{ADC}_{\text {pre-RT}}$, with significant differences. $\mathrm{ADC}_{\text {lm-post-RT initially }}$ increased and changed little to $\mathrm{ADC}_{3 \mathrm{~m}-\mathrm{post}-\mathrm{RT}}, \mathrm{ADC}_{6 \mathrm{~m}-\mathrm{post}-\mathrm{RT}}, \mathrm{ADC}_{9 \mathrm{~m}-\mathrm{post}-\mathrm{RT}}$, and $\mathrm{ADC}_{12 \mathrm{~m}-\mathrm{post}-\mathrm{RT}}$, then gradually declined over time. The dynamic change trends of SFR were negatively paralleled to those of ADC, while that of XQ-sum was similar. Dose-response relationships were detected between salivary gland mean RT dose and $\triangle \mathrm{ADC}$. In PGs, negative correlations between $\Delta \mathrm{sSFR}_{9 \mathrm{~m}-\mathrm{post}-\mathrm{RT}}$ and $\Delta \mathrm{ADC}_{9 \mathrm{~m} \text {-post-RT}}$, and $\Delta \mathrm{SSFR}_{12 \mathrm{~m}-\text { post-RT }}$ and $\Delta \mathrm{ADC}_{12 \mathrm{~m} \text {-post-RT }}$ were detected. In SMGs, negative correlations between $\Delta \mathrm{sSFR} \mathrm{R}_{12 \mathrm{~m} \text {-post-RT }}$ and $\triangle \mathrm{ADC}_{12 \mathrm{~m} \text {-post-RT, }}$, and $\Delta \mathrm{uSFR}_{12 \mathrm{~m}-\text { post-RT }}$ and $\Delta \mathrm{ADC}_{12 \mathrm{~m} \text {-post-RT }}$ were also detected. The $\mathrm{ADCs}$ of patients with severe subjective xerostomia were significantly higher, while patients with moderate subjective xerostomia presented a tendency toward higher ADCs compared to those with mild xerostomia from 6 to 12 months post RT.
\end{abstract}

The authors W.-j. Fan and F. Teng contributed equally to the manuscript.
Wen-jun Fan
answer.cool@163.com
Feng Teng
tfeng0611@126.com
Yan-rong Luo
9322453@qq.com
Wei Yu
yuwei@126.com
Qian Zhang
1098927835@qq.com

Yi-ping Lu

1052474357@qq.com

Lin $\mathrm{Ma}, \mathrm{PhD}$

malinpharm@sina.com

1 Medical School of Chinese PLA, No. 28 Fuxing Road, 100853 Beijing, China

2 Armed Police Forces Corps Hospital of Henan Province, No. 1 Kangfu Road, 450052 Zhengzhou, China

3 Department of Radiation Oncology, China-Japan Friendship Hospital, No. 2 Yinghuayuan Dongjie, 100029 Beijing, China

4 Department of Radiation Oncology, First Medical Center of Chinese PLA General Hospital, No. 28 Fuxing Road, 100853 Beijing, China 
Conclusion As part of routine follow-up MRI in NPC patients, DWI might be a promising modality for follow-up assessing the dynamic changes of major salivary gland function and might be more powerful in the late post-RT period.

Keywords Diffusion-weighted imaging $\cdot$ Radiotherapy $\cdot$ Xerostomia $\cdot$ Salivary gland dysfunction $\cdot$ Nasopharyngeal carcinoma

\section{Introduction}

Nasopharyngeal carcinoma (NPC) is one of the most common malignancies in southern China and southeast Asia, with an extremely high geographical incidence of about 25-30 per 100,000 persons per year [1, 2]. Radiotherapy (RT) alone or in combination with chemotherapy is the primary radical treatment for NPC patients and provides high locoregional control. Major salivary glands such as parotid glands (PGs) and submandibular glands (SMGs) are both highly radiosensitive and often involved in or adjacent to RT targets designed for NPC and apt to injury even after low-dose radiation [3]. Radiation-induced salivary gland injury and consequences such as xerostomia or dry mouth are probably the most common persistent oral sequelae for NPC patients who receive therapeutic doses of RT. Patients with xerostomia have considerable difficulties with chewing and swallowing and are impaired in speech and social interactions, which have further implications with respect to quality of life, especially in long-term survivors [4-6]. The probability and severity of xerostomia mostly depends on the dose distributions in PGs and SMGs [7]. Lower radiation doses lead to a better-maintained salivary flow after RT. Management of radiation-induced xerostomia by, e.g., pharmacological interventions (e.g., amifostine, pilocarpine) or submandibular gland transfer, appears to be beneficial with insufficient evidence. These treatments are not routinely recommended due to potential adverse effects of pharmacological agents and treatment delay of invasive submandibular gland transfer [8]. Therefore, the key lies in prevention by radiation dose reduction in major salivary glands.

During the past two decades, high-precision intensitymodulated radiation therapy (IMRT) technology with better conformity and dose homogeneity, such as helical tomotherapy (HT), has been increasingly applied for NPC treatment. With IMRT, substantial dose reductions could be achieved to major salivary glands, resulting in retention of salivary output and amelioration of xerostomia without compromising dose delivery to the tumor [9-11]. But despite the emergence of HT, the incidence of xerostomia 1 year and 2 years post RT in NPC patients is still at a high level. Based on this, long-term follow-up evaluation of the dynamic physiological changes of irradiated salivary glands would be important to better understand the mechanism of radiation-induced injury, which in turn aids the investiga- tion of methods to mitigate symptoms of xerostomia such as optimization of treatment plans before implementing RT.

Traditionally, radiation-induced salivary gland dysfunction can be assessed subjectively according to a patient's symptoms as well as objectively using quantified saliva production or excretion (scintigraphy and/or saliva flow) [12]. Salivary gland scintigraphy (SGS) has been shown to be reliable in evaluating salivary gland function [13, 14]. But the usefulness of SGS is limited by its low spatial resolution, the complex procedure, and its involvement of ionizing radiation. Salivary flow rate (SFR) measurement is the most commonly applied objective method in the clinic to quantify the severity of irradiated salivary gland dysfunction, but its results do not depict any morphological or physiological change of the irradiated salivary glands [12].

Diffusion-weighted imaging (DWI), which can assess and quantify the structural and pathophysiological changes by visualizing the random thermal motion of water molecule diffusion, has been widely used as one of the routine follow-up MR examinations of NPC patients for decades. In recent years, initial studies [15-19] of DWI in assessing radiation-induced salivary gland dysfunction have been documented and observed significant alterations of apparent diffusion coefficients (ADCs), the quantitative parameter of DWI, of major salivary glands pre and post RT in NPC patients. Several of these studies [17-19] further found a correlation between pre- and post-RT changes of $\mathrm{ADC}\left(\triangle \mathrm{ADC} \mathrm{C}_{\text {post-pre }}\right)$ and volume atrophy of PGs as well as ratios of salivary excretion fraction (rEF), the quantitative parameter of SGS. But until now, most of these initial studies have merely focused on the evaluation of PG dysfunction, whereas severity of xerostomia does not only attribute to the injury of PG but also SMG [20, 21]. Meanwhile, nearly all previous relative studies only compared ADCs of major salivary glands at one timepoint after RT with that before RT, continuous observations are scarce. And the correlations between changes in ADC and clinical parameters, such as subjective xerostomia degree and SFR, remain unclear. Besides, to the best of our knowledge, studies assessing salivary gland dysfunction with DWI in NPC patients treated with HT have not been documented. It would be interesting to investigate the radiation-induced physiological changes of irradiated PGs and SMGs at a lower radiation dose by using HT.

Therefore, the purpose of this preliminary study was to observe the dynamic changes in ADCs of both PGs 
and SMGs in NPC patients 1 year post HT, to analyze whether alterations of PG and/or SMG ADCs correlated with mean radiation dose and changes in SFR under stimulated/unstimulated conditions at six different follow-up timepoints, to further compare ADCs among patients with different subjective degrees of xerostomia.

\section{Materials and methods}

\section{Patients}

This prospective study registered with number ChiCTR1900024328 in the Chinese Clinical Trial Registry was approved by the research ethics board of the Chinese PLA General Hospital. Written informed consent was obtained from all eligible patients prior to participation.

From July 2017 to January 2018, 31 consecutive patients with pathologically confirmed NPC who were scheduled for bilateral neck HT with curative intent were prospectively recruited in this study. All patients had a good performance status (ECOG 0-1) and were scheduled to undergo MR evaluation and follow-ups at our hospital. None of them had received prior RT or surgery to the head and neck region, any previous salivary gland diseases such as Sjogren's syndrome, any other medical causes of xerostomia, MRI contraindications, or any metal implants in the mouth. All patients underwent six MR examinations with an identical scan protocol each time, namely 2-3 weeks before RT (pre-HT) and 1, 3, 6, 9, and 12 months after completion of RT (1m-post-RT, 3m-post-RT, 6m-post-RT, 9m-post-RT, $12 \mathrm{~m}$-post-RT). Meanwhile, measurement of SFR as well as assessment of subjective degree of xerostomia were conducted an hour before each MR examination.

\section{Treatment}

All patients were treated with induction chemotherapy followed by concurrent chemoradiotherapy. A two-cycle TP or TPF regimen was used in induction chemotherapy, i.e., docetaxel (T) $70 \mathrm{mg} / \mathrm{m}^{2}$ on day 1 and cisplatin (P) $40 \mathrm{mg} / \mathrm{m}^{2}$ on days 1 and 2; for patients with massive tumors at the primary site, 5 -fluorouracil (F) $700 \mathrm{mg} / \mathrm{m}^{2}$ on days $1-5$ was added accordingly. Concomitant chemotherapy used docetaxel $70 \mathrm{mg} / \mathrm{m}^{2}$ or cisplatin $70 \mathrm{mg} / \mathrm{m}^{2}$ for three cycles every 21 days. RT was performed with HT (TomoTherapy; Accuray Inc., Sunnyvale, CA, USA) to the nasopharyngeal lesions, metastatic lymphadenopathy, and the neck lymphatic drainage areas. The total prescribed doses within the nasopharyngeal lesions and metastatic lymphadenopathy were $67.5 \mathrm{~Gy}$ with $2.25 \mathrm{~Gy}$ per fraction, while that within highrisk areas was $60 \mathrm{~Gy}$ with $2.0 \mathrm{~Gy}$ per fraction, and in lowrisk areas $54 \mathrm{~Gy}$ with $1.8 \mathrm{~Gy}$ per fraction. The range of high- risk area and low-risk area was determined by the extent of nasopharyngeal lesions as well as the region of lymph node metastasis. RT was delivered once daily, five fractions per week, with a total of 30 fractions for six weeks. Treatment planning was optimized on the Pinnacle 3.8.0 treatment workstation (Philips Medical Systems, Fitchburg, WI, USA) by the reverse intensity-modulated planning system. All subjects were scheduled for a salivary gland-sparing technique to keep radiation doses to bilateral PGs and SMGs as low as possible on the promise of meeting the prescription dose coverage of $95 \%$ or more to the treatment volume. The mean radiation dose constraints for bilateral PGs were no more than $28 \mathrm{~Gy}$, so do SMG-spared (ipsilateral level Ib out of high-risk area). No salivary protectors or stimulants were allowed during therapy or the 1-year study follow-up period. Details of plan design and dose-volume constraints for organs at risk referred to a previous article of our center [22].

\section{MRI protocol}

All MRI scans were performed on a 3.0-T MR system with a maximum gradient capability of $23 \mathrm{mT} / \mathrm{m}$ (Signa HDx, GE Healthcare, Milwaukee, WI, USA). Patients were imaged in the supine position using a 16-channel neurovascular head and neck array coil. For morphologic evaluation, an axial T1-weighted spin-echo sequence (TR/TE, $428 / 11.6 \mathrm{~ms}$ ) was performed using a matrix of $288 \times 192$, a field of view (FOV) of $180 \times 240 \mathrm{~mm}$, and a section thickness of $5 \mathrm{~mm}$ with an intersection gap of $1 \mathrm{~mm}$. Then, fast spin-echo T2-weighted (TR/TE, 3000/59.5 ms) with fat suppression was obtained in the transverse plane with a matrix of $320 \times 224$, an FOV of $180 \times 240 \mathrm{~mm}$, and a section thickness of $5 \mathrm{~mm}$ with an intersection gap of $1 \mathrm{~mm}$. This sequence was performed as a reference for the following DWI images to improve the delineation of both PGs and SMGs. The images encompassed the area from the skull base to the level of the glottis, including the full volume of PGs and SMGs.

Thereafter, an axial echoplanar DWI sequence was performed with a TR of $5000 \mathrm{~ms}$, a minimum TE of $74.6 \mathrm{~ms}$, a matrix of $128 \times 128$, and excitations. The other parameters, including FOV, section thickness, and intersection spacing, were identical to those used for T2-weighted imaging. The $b$ values used were $0,600 \mathrm{~s} / \mathrm{mm}^{2}$. The motion-probing gradients were placed on the three orthogonal directions with the same strength. Eleven to thirteen sections were obtained, and the acquisition time of the DWI sequence was about $2 \mathrm{~min}$. 


\section{Image analysis}

The data were digitally transferred from the MRI unit console to an independent Linux workstation with dedicated software (Advantage Workstation version 4.3, GE Healthcare). ADC maps were automatically constructed for DWI images by pixel-by-pixel calculation. ADC values were calculated using a mono-exponential model: $S_{i}=S_{0} \times \exp \left(-b_{i} \times \mathrm{ADC}\right)$, where $S_{i}$ is the signal intensity measured on the $i$-th $b$ value image, $b_{i}$ is the corresponding $b$ value, and $S_{0}$ indicates the exact (without noise) signal intensity for $b=0 \mathrm{~s} / \mathrm{mm}^{2}$.

All the MR images were independently analyzed and measured by two radiologists with more than 8 years of experience in head and neck MR imaging, who were blinded to all clinical information. The regions of interest (ROIs) were manually drawn on the largest three contiguous slices of ADC images of bilateral PGs and SMGs to encompass as much of the gland parenchyma as possible in reference to T2-weighted images. The regions containing large vessels, such as the retromandibular vein and external carotid artery were excluded. The final ADC value of each salivary gland was defined as the mean value of three slices. The change rates of ADCs were calculated using the following equation:

$$
\begin{aligned}
\Delta \mathrm{ADC}_{(i) \mathrm{m}-\text { post-RT }}= & \left(\mathrm{ADC}_{(i) \mathrm{m}-\text { post-RT }}-\mathrm{ADC}_{\text {pre-RT }}\right) / \\
& \mathrm{ADC}_{\text {pre-RT }} \times 100 \%
\end{aligned}
$$

Where $(i)$ m-post-RT represents the exact month after completion of RT, $\triangle \mathrm{ADC}_{(i) \mathrm{m} \text {-post-RT }}$ is the change rate of $\mathrm{ADC}$ value at $(i) \mathrm{m}$-post-RT compared with pre-RT, and $\mathrm{ADC}_{\text {pre-RT }}$ is the ADC value before RT.

The final results were recorded as the mean value of two observers' measurements. ADC values of both PGs and SMGs were repeatedly measured by one radiologist at an interval of 4 weeks to evaluate the intra-observer reproducibility.

\section{Whole-mouth SFR measurement}

Whole-mouth SFR measurement, one kind of sialometry, directly measures the function of salivary glands. In our study, whole-mouth salivary output measurement was conducted using the spitting method. Patients were instructed to spit their saliva in a graded tube for $5 \mathrm{~min}$, both at rest and upon stimulation by dipping citric acid of $2 \%$ concentration on the tongue tip with a cotton bud once every $20 \mathrm{~s}$. The collected saliva volume in the two conditions was recorded and then used to calculate SFR under unstimulated (uSFR) as well as SFR under stimulated conditions (sSFR) as well as their change rates using the following equation:

$$
\begin{aligned}
\Delta \mathrm{SFR}_{(i) \mathrm{m}-\text { post-RT }}= & \left(\mathrm{SFR}_{(i) \mathrm{m}-\text { post-RT }}-\mathrm{SFR}_{\text {pre-RT }}\right) / \\
& \mathrm{SFR}_{\text {pre-RT }} \times 100 \%
\end{aligned}
$$

Where $\Delta \mathrm{SFR}_{(i) \mathrm{m} \text {-post-RT }}$ is the change rate of SFR at $(i) \mathrm{m}$ post-RT compared with pre-RT, and SFR $\mathrm{Rre}_{\mathrm{RT}}$ is SFR before RT.

Patients were asked not to eat or drink for at least $1 \mathrm{~h}$ before the examination.

\section{Assessment of the degree of xerostomia}

Before each SFR measurement, patients were asked to complete a modified xerostomia-specific questionnaire which has been validated previously [23]. Briefly, the questionnaire consists of five items regarding dryness when eating, speaking, swallowing, and chewing, and five relating to dryness while at rest. Each item is rated on a four-point ordinal scale from 0 to 3 , with higher scores indicating more severe xerostomia. The score of each item is summed up to produce a summary score ranging from 0 to 30 . The severity of xerostomia is classified by the summary score (XQ-sum): mild xerostomia, XQ-sum $\leq 10$ points; moderate xerostomia, $10<\mathrm{XQ}$-sum $\leq 20$ points; severe xerostomia, XQ-sum $>20$ points.

\section{Statistical analysis}

Statistical analysis was performed with SPSS 24.0 Software (IBM Corp., Armonk, NY, USA). Descriptive statistics were used to analyze patients' demographic data and clinical characteristics. Numerical data were presented as the mean \pm standard deviation. Kolmogorov-Smirnov's test was used to determine whether the parameters were normally distributed. Paired two-tailed Student's $t$-tests were used to compared mean radiation doses to PGs and SMGs, ADCs of PGs and SMGs, and SFR under different conditions at each timepoint. The dynamic changes of ADCs and SFR, and the differences in ADCs among different degrees of xerostomia were analyzed by repeated measures analysis or one-way analysis of variance with the Bonferroni post-test. Correlations between $\triangle \mathrm{ADC}$ and $\triangle \mathrm{SFR}$ and mean radiation doses were investigated using Pearson's correlation test. The intra- and inter-observer reproducibility of ADC values were analyzed using the intraclass correlation coefficient (ICC). Two-sided $P$-values less than 0.05 were considered as statistically significant. 


\section{DWI images of PGs ADC images of PGs DWI images of SMGs ADC images of SMGs}
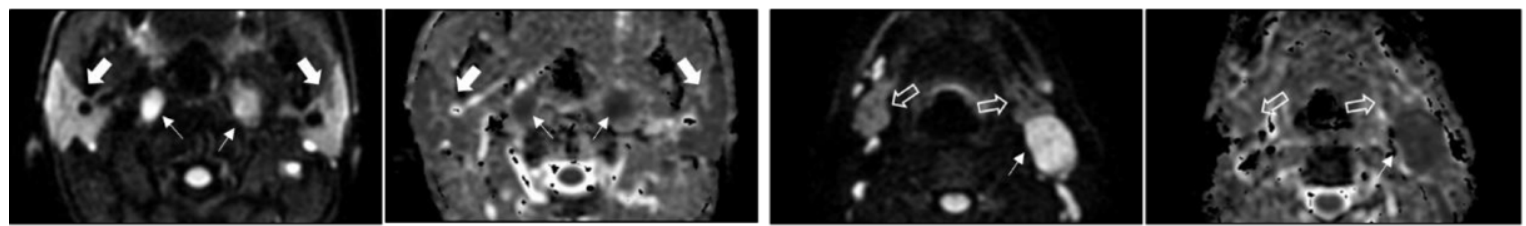

Pre-RT
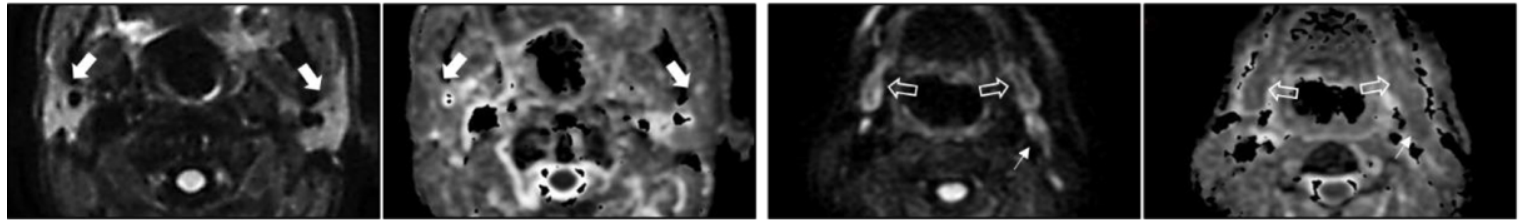

1m-post-RT
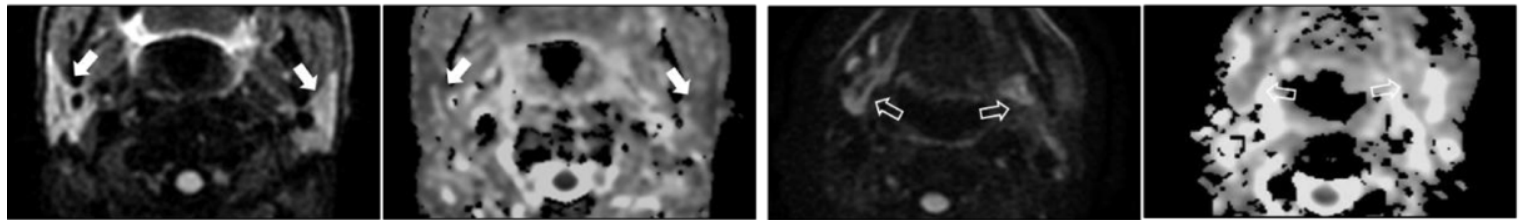

3m-post-RT
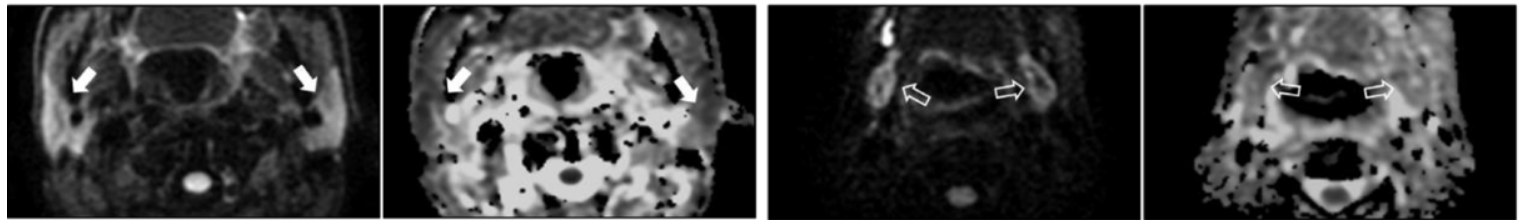

6m-post-RT
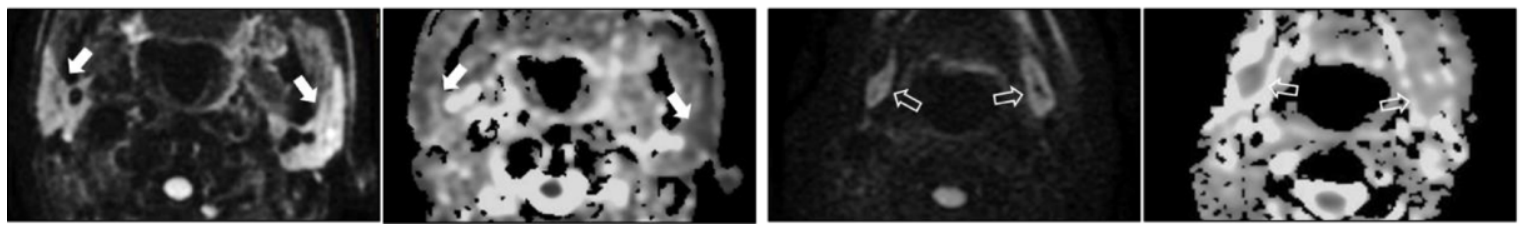

12m-post-RT

Fig. 1 Diffusion weighted imaging (DWI) and apparent diffusion coefficient (ADC) images of bilateral parotid glands (PGs; white solid arrow) and submandibular glands (SMGs; white hollow arrow) of a 49-year-old male NPC patient at different timepoints pre and post RT. For both PGs and SMGs, ADC images illustrated a relatively slightly higher signal at 1m-post-RT and 3m-post-RT compared to that of pre-RT, with a gradually decreasing signal at $6 \mathrm{~m}$-post-RT and $12 \mathrm{~m}$-post-RT. Meanwhile, the metastatic lymph nodes (white arrow) apparently disappeared after $1 \mathrm{~m}$-post RT

\section{Results}

All subjects successfully underwent the whole therapy and follow-up MR examinations. No measurement was excluded because of insufficient quality. The pre- and postRT DWI and ADC images of bilateral PGs and SMGs of one representative subject are shown in Fig. 1. Excellent reproducibility of the measurement of ADC for both PGs and SMGs was achieved, with the inter-observer ICC at $0.92(P<0.001)$ and the intra-observer ICC at 0.94 $(P<0.001)$. The main characteristics of subjects and tumor are presented in Table 1.

\section{Comparison of PGs and SMGs}

There was no difference in mean radiation dose to bilateral PGs or SMGs, whereas the mean radiation dose to SMGs was significantly higher than to PGs $(P<0.001)$, as shown in Table 1. At each timepoint, ADCs of bilateral PGs were similar to each other, the same was true for bilateral SMGs, while ADCs of PGs were significantly lower than those of SMGs $(P<0.05)$, as shown in Table 2.

\section{Dynamic changes of ADC at different follow-up timepoints}

The change trends over time of the ADC of PGs and SMGs were similar, as shown in Fig. 2. With either PGs or SMGs, $\mathrm{ADC}_{\mathrm{post}-\mathrm{RT}}$ were all higher than $\mathrm{ADC}_{\text {pre-RT }}$, with significant differences $(P \leq 0.01) ; \mathrm{ADC}_{1 \mathrm{~m}-\mathrm{post}-\mathrm{RT}}$ and $\mathrm{ADC}_{3 \mathrm{~m} \text {-post-RT }}$ were nearly equal $(P=1.000) ; \mathrm{ADC}_{6 \mathrm{~m} \text {-post-RT }}$, $\mathrm{ADC}_{9 \mathrm{~m} \text {-post-RT }}, \mathrm{ADC}_{12 \mathrm{~m} \text {-post-RT became lower and lower over }}$ time $(P<0.05)$. But for SMGs, the statistical differences between $\mathrm{ADC}_{6 \mathrm{~m}-\mathrm{post}-\mathrm{RT}}$ and $\mathrm{ADC}_{9 \mathrm{~m}-\mathrm{post}-\mathrm{RT}}$, and $\mathrm{ADC}_{9 \mathrm{~m}-\mathrm{post}-\mathrm{RT}}$ 
Table 1 Clinical characteristics of patients and tumors

\begin{tabular}{ll}
\hline Characteristic & Value \\
\hline Patients (male/female) & $31(27 / 4)$ \\
Age (years) & \\
Mean (range) & $48.6(18-67)$ \\
T stage & \\
T1 & $4(12.9 \%)$ \\
T2 & $15(48.4 \%)$ \\
T3 & $6(19.4 \%)$ \\
T4 & $6(19.4 \%)$ \\
N stage & \\
N1 & $2(6.5 \%)$ \\
N2 & $23(74.2 \%)$ \\
N3 & $6(19.4 \%)$ \\
Clinical stage & \\
Stage II & $1(3.2 \%)$ \\
Stage III & $20(64.5 \%)$ \\
Stage IVA & $10(32.3 \%)$ \\
Mean radiation dose (Gy) & \\
PG-R & $30.62 \pm 2.91(27.10-40.18)$ \\
PG-L & $29.80 \pm 2.47(26.70-37.79)$ \\
SMG-R & $46.64 \pm 16.61(24.47-65.59)$ \\
SMG-L & $41.27 \pm 16.86(22.38-66.35)$ \\
\hline$P$ - $L$ left &
\end{tabular}

$P G-L$ left parotid gland, $P G-R$ right parotid gland, $S M G-L$ left submandibular gland, $S M G-R$ right submandibular gland

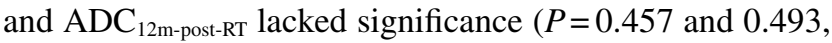
respectively).

\section{Dynamic changes of SFR at different follow-up timepoints}

At each timepoint, sSFR was higher than uSFR $(P<0.001$ for all), as shown in Table 2. For both sSFR and uSFR, $\mathrm{SFR}_{1 \mathrm{~m}-\mathrm{post}-\mathrm{RT}}$ apparently declined from $\mathrm{SFR}_{\text {pre-RT}}$, changed

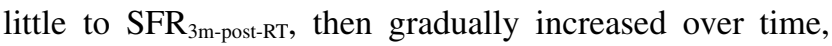

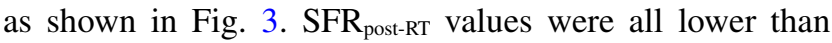
$\mathrm{SFR}_{\text {pre-RT}}$, with the exception that the difference between $\mathrm{SFR}_{12 \mathrm{~m} \text {-post-RT }}$ and $\mathrm{SFR}_{\text {pre-RT }}$ was not statistically significant ( $P=0.737$ for sSFR, $P=0.738$ for uSFR). The differences in SFR between two continuous timepoints post RT were not significant $(P>0.05$ for all). But there were significant differences between $\mathrm{SFR}_{12 \mathrm{~m} \text {-post-RT }}$ and $\mathrm{SFR}_{3 \mathrm{~m}-\mathrm{post}-\mathrm{RT}}$ of $\mathrm{SSFR}$ $(P=0.022)$, and $\mathrm{SFR}_{12 \mathrm{~m} \text {-post-RT }}$ and $\mathrm{SFR}_{6 \mathrm{~m} \text {-post-RT }}$ of uSFR $(P=0.013)$.

\section{Correlation of $\triangle A D C$ with RT dose as well as $\triangle \mathrm{SFR}$}

As shown in Table 3, linear correlations were found between mean radiation dose to $\mathrm{PGs}$ and $\triangle \mathrm{ADC}_{\mathrm{post} \text {-pre, and }}$ the dose-response relationship was seen at each followup timepoint post RT. Linear correlations were also found between mean radiation dose to $\mathrm{SMGs}$ and $\triangle \mathrm{ADC}_{6 \mathrm{~m}-\mathrm{post}-\mathrm{RT}}$, $\triangle \mathrm{ADC}_{9 \mathrm{~m} \text {-post-RT, }}$, and $\Delta \mathrm{ADC}_{12 \mathrm{~m} \text {-post-RT. }}$ There was a significant negative correlation between $\Delta \mathrm{sSFR}_{9 \mathrm{~m}-\text { post-RT }}$ and $\Delta \mathrm{ADC}_{9 \mathrm{~m}-\mathrm{post}-\mathrm{RT}}(r=-0.369, P=0.040)$, and $\Delta \mathrm{sSFR}_{12 \mathrm{~m} \text {-post-RT }}$ and $\triangle \mathrm{ADC}_{12 \mathrm{~m} \text {-post-RT }}(r=-0.411, P=0.021)$ of PGs, between $\Delta \mathrm{SSFR}_{12 \mathrm{~m} \text {-post-RT }}$ and $\triangle \mathrm{ADC}_{12 \mathrm{~m} \text {-post-HT }}(r=-0.417, P=0.020)$, and $\Delta \mathrm{uSFR}_{12 \mathrm{~m} \text {-post-RT }}$ and $\Delta \mathrm{ADC}_{12 \mathrm{~m} \text {-post-RT }}(r=-0.392, P=$ 0.029) of SMGs.

\section{Relationship between ADC and subjective degree of xerostomia}

As shown in Fig. 4, the overall change trend post RT of XQsum was in line with that of ADC. From 3m-post-RT, XQsum gradually decreased over time, with XQ-sum $12 \mathrm{~m}$-post-RT apparently lower. Classified by XQ-sum, the percentage of patients with mild, moderate, and severe xerostomia at $1 \mathrm{~m}$-post-RT was $35.5 \%, 48.4 \%$, and $16.1 \%$, respectively, while at $12 \mathrm{~m}$-post-RT, the percentage of patients with mild xerostomia increased to $90.3 \%$, moderate xerostomia declined to $9.7 \%$, and no patient had severe xerostomia. With further analysis, $\mathrm{ADC}_{1 \mathrm{~m} \text {-post-RT }}$ and $\mathrm{ADC}_{3 \mathrm{~m}-\text { post-RT }}$ of PGs and $\mathrm{ADC}_{1 \mathrm{~m} \text {-post-RT }}$ of SMGs in patients with mild and moderate xerostomia were significantly higher than in those with severe xerostomia $(P<0.05$ for both). Compared to patients with mild xerostomia, $\mathrm{ADC}_{1 \mathrm{~m} \text {-post-RT }}$ and $\mathrm{ADC}_{3 \mathrm{~m} \text {-post-RT }}$ of $\mathrm{PGs}$ and SMGs in patients with moderate xerostomia showed no significant differences, but $\mathrm{ADC}_{6 \mathrm{~m}-\mathrm{post}-\mathrm{RT}}, \mathrm{ADC}_{9 \mathrm{~m} \text {-post-RT}}$, and

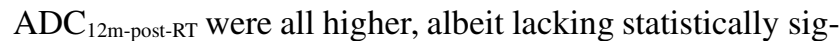
nificant differences $(P>0.05$ for all), as shown in Fig. 5 .

\section{Discussion}

Xerostomia is defined by the Common Toxicity Criteria of Adverse Events (CTCAEs) as a disorder characterized by reduced salivary flow in the oral cavity. PGs and SMGs are the main sources of saliva flow. Stimulated salivary production is largely derived from PGs (approximately 60\% of total saliva in stimulated conditions), while resting or unstimulated saliva is mostly produced by SMGs (nearly $90 \%$ of total saliva in unstimulated conditions) [24]. Radiation-induced xerostomia is mostly attributed to injury of PGs and SMGs. To accurately evaluate post-RT changes of salivary gland function or xerostomia, different assessment methods have been reported in the literature [12, 24]. Our study using DWI to assess dynamic changes of salivary gland function over time in 1-year post-RT and particularly to assess PGs and SMGs separately, found a strong correlation between pre- and post-RT changes of ADC and those of SFR under unstimulated/stimulated conditions as well as with the degree of subjective xerostomia. To the best of our knowledge, this is the first follow-up research so far 

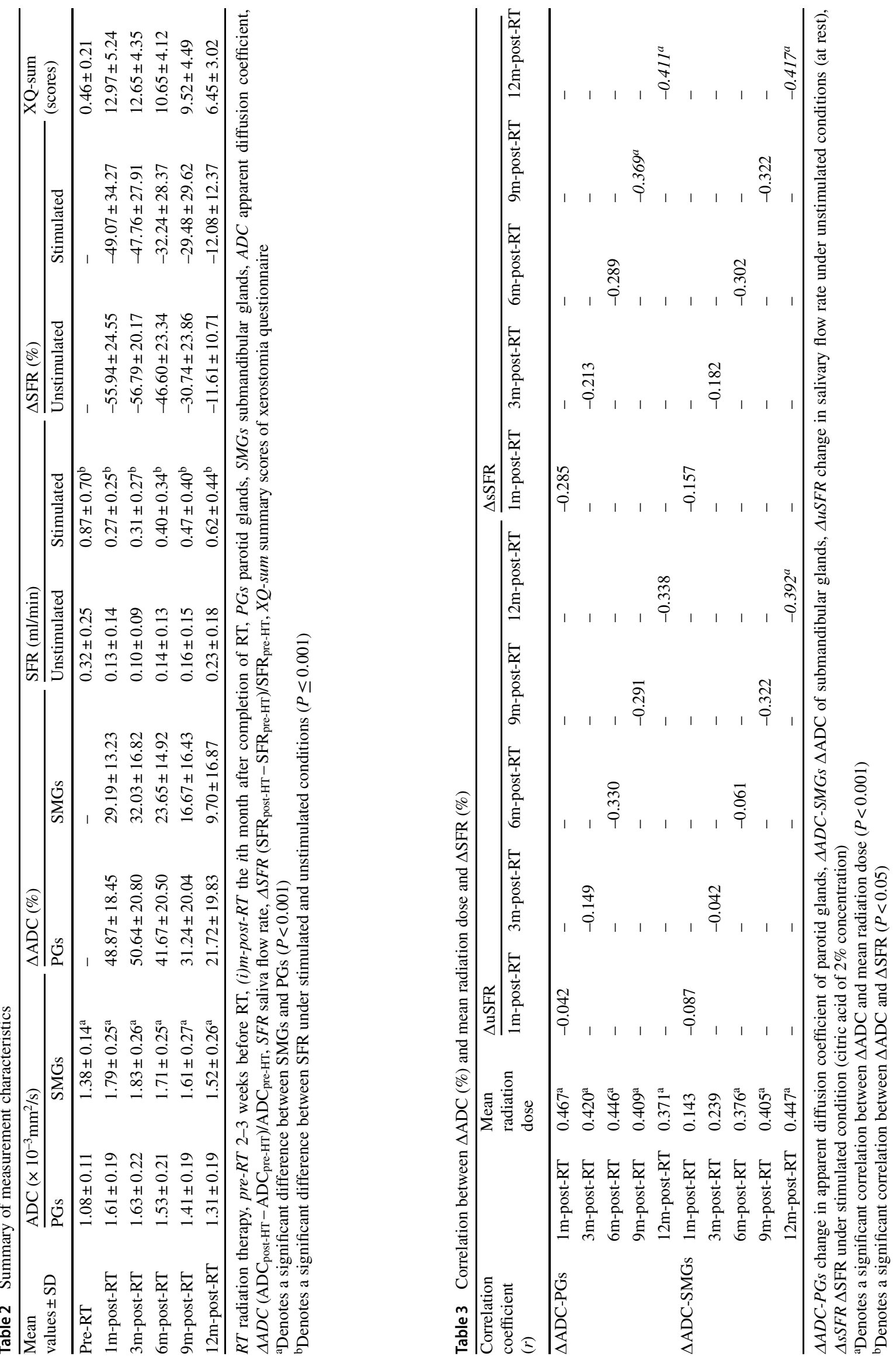
Fig. 2 Dynamic changes of ADC of both parotid glands $(P G s)$ and submandibular glands $(S M G s)$. Line graphs indicate a similar change trend over time for both PGs and SMGs, i.e., ADC increased significantly $1 \mathrm{~m}$-post-RT, followed by no obvious change until $3 \mathrm{~m}$-post-RT, and then gradually declined

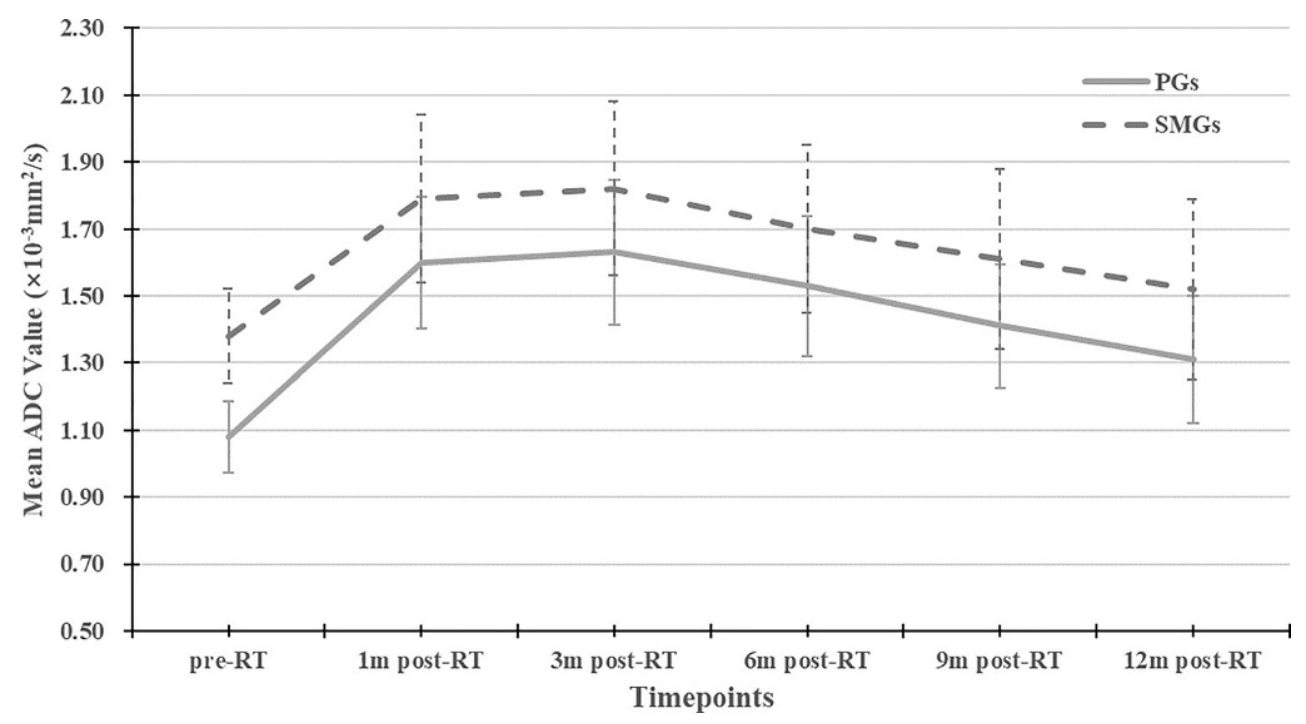

Fig. 3 Dynamic changes of SFR under unstimulated (uSFR) and stimulated conditions (sSFR). Line graphs indicate a similar change trend over time for both USFR and sSFR, i.e., SFR decreased significantly $1 \mathrm{~m}$ post-RT, followed by no obvious change until 3m-post-RT, and then gradually increased

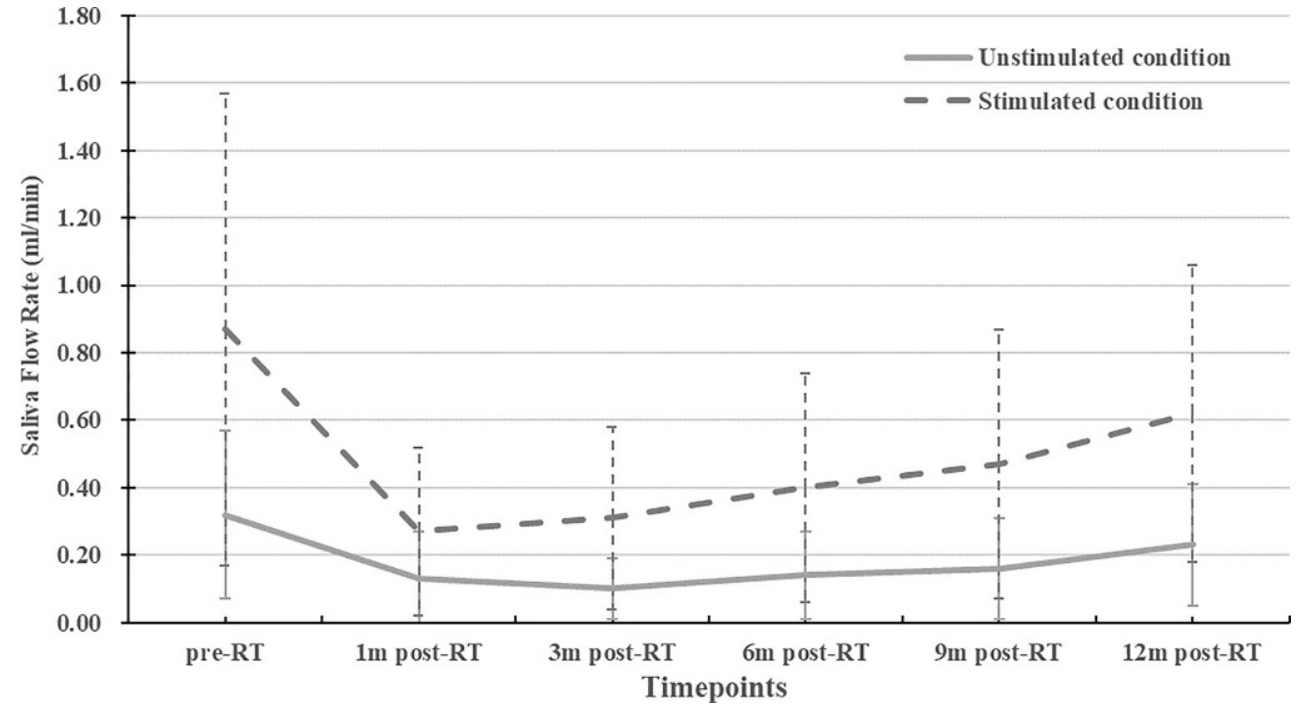

to explore the correlations of DWI parameters with clinical parameters containing objective SFR measurement and subjective degree of xerostomia simultaneously.

PGs and SMGs are composed of acinar cells, which produce saliva. Animal studies $[25,26]$ found that both irradiated PGs and SMGs were characterized by acinar cell loss or atrophy, interstitial fibrosis, and so forth. In our study, at each timepoint either pre or post RT, ADCs of SMGs were significantly higher than of PGs. This finding is in accordance with previous studies $[18,19]$ and it is thought to reflect the much higher proportional amount of extracellular water of SMG. Compared to $\mathrm{ADC}_{\text {pre-RT }}, \mathrm{ADC}_{\text {post-RT }}$ rise significantly. This rise is thought to reflect the increased water diffusivity in injured salivary glands as a result of acinar cellular loss. After 3-months post RT, ADC became lower and lower over time. The drop of ADC is thought to reflect the decreased water diffusivity owing to the increase of acinar cell number, which indicates functional recovery of salivary glands. A previous rat model study [25] observed an initial drop of acinar cell number of PGs followed by an increase in the late post-irradiation period (120-240 days).

Regarding the dosage effect, the relationship between radiation dose absorbed by salivary glands and $\triangle \mathrm{ADC}_{\text {post-pre }}$ has previously been investigated. In their series of 34 NPC patients, Marzi et al. [27] found no significant correlation between the RT dose and ADC changes of PGs from measurement timepoints at half way through and at the end of RT. Loimu et al. [18] found a dose-response correlation between radiation dose to PGs as well as SMGs and $\triangle \mathrm{ADC}_{\text {post-pre }}$ from measurement timepoints prior to RT onset and at a mean of 6 months post RT. Our study further showed significantly positive correlations between radiation dose and $\triangle \mathrm{ADC}_{\text {post-pre }}$ at five timepoints from 1 month to 12 months post RT of PGs and $\triangle \mathrm{ADC}_{\text {post-pre }}$ at 6,9 , and 
Fig. 4 Dynamic changes of xerostomia questionnaire summary score (XQ-sum). Line graph indicating change trend over time of XQ-sum shows an initial increase 1m-post-RT with subsequently minor change until $3 \mathrm{~m}$-post-RT and then a gradual decrease from 3m-post-RT to $12 \mathrm{~m}$-post-RT

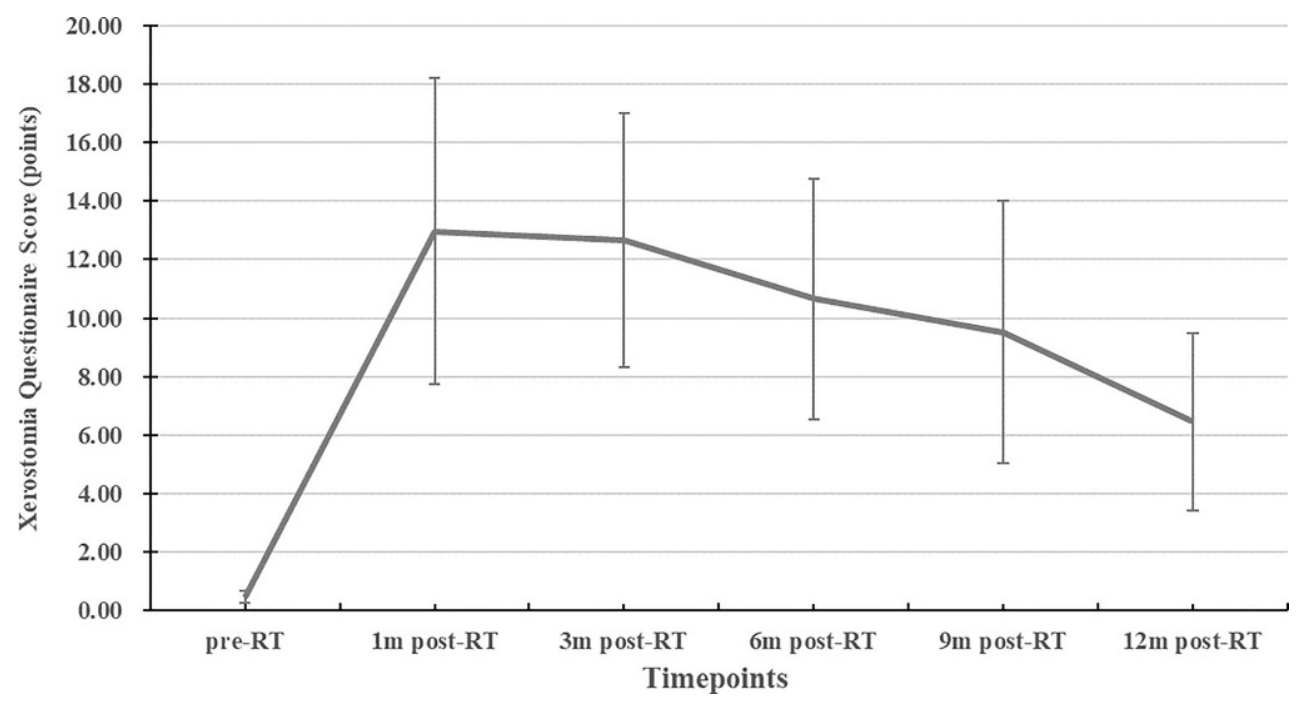

12 months post RT of SMGs, which indicates a dose-dependent loss of acinar cells and a continuous dose-response effect over time.

SFR measurement remains the benchmark for assessment of salivary gland function. It directly measures the function of salivary glands and can be classified into a whole-mouth output measurement and a selective salivary gland output measurement. The former measurement is relatively fast, noninvasive, and easily achieved, which renders it more commonly used in clinics. Our study employed the whole-mouth output measurement to acquire SFR under unstimulated and stimulated conditions. In our study, sSFR was higher than uSFR at each timepoint. The overall change trend of SFR was negatively paralleled to that of ADC; SFR declined in the first 3 months post RT, then gradually increased over time. This finding corresponds with the study of Gupta [14], who found a maximal decrease in $\mathrm{rEF}$ at $3 \mathrm{~m}$-post-RT with then functional recovery over time in a cohort of 41 head and neck cancer patients by using quantitative SGS to assess PG function.

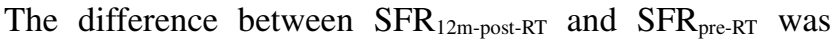
not statistically significant, but the differences between $\mathrm{SFR}_{12 \mathrm{~m} \text {-post-RT }}$ and $\mathrm{SFR}_{3 \mathrm{~m} \text {-post-RT }}$ of sSFR and $\mathrm{SFR}_{12 \mathrm{~m} \text {-post-RT }}$ and $\mathrm{SFR}_{6 \mathrm{~m} \text {-post-RT }}$ of uSFR were significant. The results indicated that both uSFR and SSFR could recover nearly to the preRT level 1-year post RT, but the time to begin recovery of sSFR was ahead of uSFR.

One unique feature of our study is the correlation between $\triangle \mathrm{ADC}$ and $\triangle \mathrm{SFR}$ under different conditions at different follow-up timepoints, which, to the best of our knowledge, has not been investigated before. In the study, significant negative correlations were found between $\Delta \mathrm{SFR}_{9 \mathrm{~m} \text {-post-RT }}$ and $\Delta \mathrm{ADC}_{9 \mathrm{~m} \text {-post-RT }}$, and $\Delta \mathrm{SFR}_{12 \mathrm{~m} \text {-post-RT }}$ and $\Delta \mathrm{ADC}_{12 \mathrm{~m} \text {-post-RT }}$ of PGs, as well as $\Delta \mathrm{SFR}_{12 \mathrm{~m} \text {-post-RT }}$ and $\triangle \mathrm{ADC}_{12 \mathrm{~m} \text {-post-RT }}$ of SMGs in the acid-stimulated condition and $\Delta \mathrm{SFR}_{12 \mathrm{~m} \text {-post-RT }}$ and $\Delta \mathrm{ADC}_{12 \mathrm{~m} \text {-post-RT }}$ of SMGs in the resting condition. The correlations were in accordance with physiological function of saliva production as mentioned above: both PGs and SMGs produce the majority of saliva during stimulation, while SMGs alone produce the majority of saliva while at rest. Furthermore, the correlations manifest that DWI can not only evaluate salivary gland dysfunction post RT separating PGs from SMGs, but also be quite suitable for assessing it in the late post-RT period.

Evaluation of the degree of xerostomia is also widely used in assessing salivary gland dysfunction clinically, and can be classified into operator-rated outcomes according to classification systems such as CTCAE, the Radiation Therapy Oncology Group (RTOG) toxicity criteria, and patientrated outcomes using questionnaires [28, 29]. In a cohort of 23 NPC patients receiving IMRT, Zhang et al. [19] found no differences in ADCs between patients with grade 1 and grade 2 xerostomia classified according to the RTOG toxicity criteria. But recent studies [30] have found that operator-rated outcomes might underestimate the actual degree of xerostomia and patient-rated outcomes are uniquely able to provide actual information. Another unique feature of our study is comparison of ADCs among different degrees of xerostomia graded by patient-rated outcomes at different follow-up timepoints. The questionnaire used in the study was the modified edition of a xerostomia-specific questionnaire which has been validated previously [23, 31]. The classification of xerostomia severity based on XQ-sum correlated to the grading system of dry mouth of CTCAE version 5.0 on the whole, with mild, moderate, and severe xerostomia equal to grade 1,2 , and 3 dry mouth by CTCAE version 5.0, respectively. The overall change trend of XQ summary scores was in keeping with that of ADC, which indicates the consistence of ADC changes and subjective xerostomia over time after RT. In early post-RT pe- 
Fig. 5 Comparison of ADC of PGs (a) and SMGs (b) among patients with different degrees of xerostomia classified by XQsum (mild xerostomia, XQ-sum $\leq 10$ points; moderate xerostomia, $10<\mathrm{XQ}$-sum $\leq 20$ points; severe xerostomia, XQsum $>20$ points). Boxplots among different degrees of xerostomia show higher ADCs in patients with severe xerostomia in the early post-RT period (1mpost-RT) and a tendency toward higher ADCs in patients with moderate xerostomia compared to those with mild xerostomia in the late post-RT period (from 6m-post-RT to $12 \mathrm{~m}$-post-RT) for both PGs and SMGs, despite a lack of statistical difference (*denote a significant difference compared to severe xerostomia; i.e., $P<0.05$ )
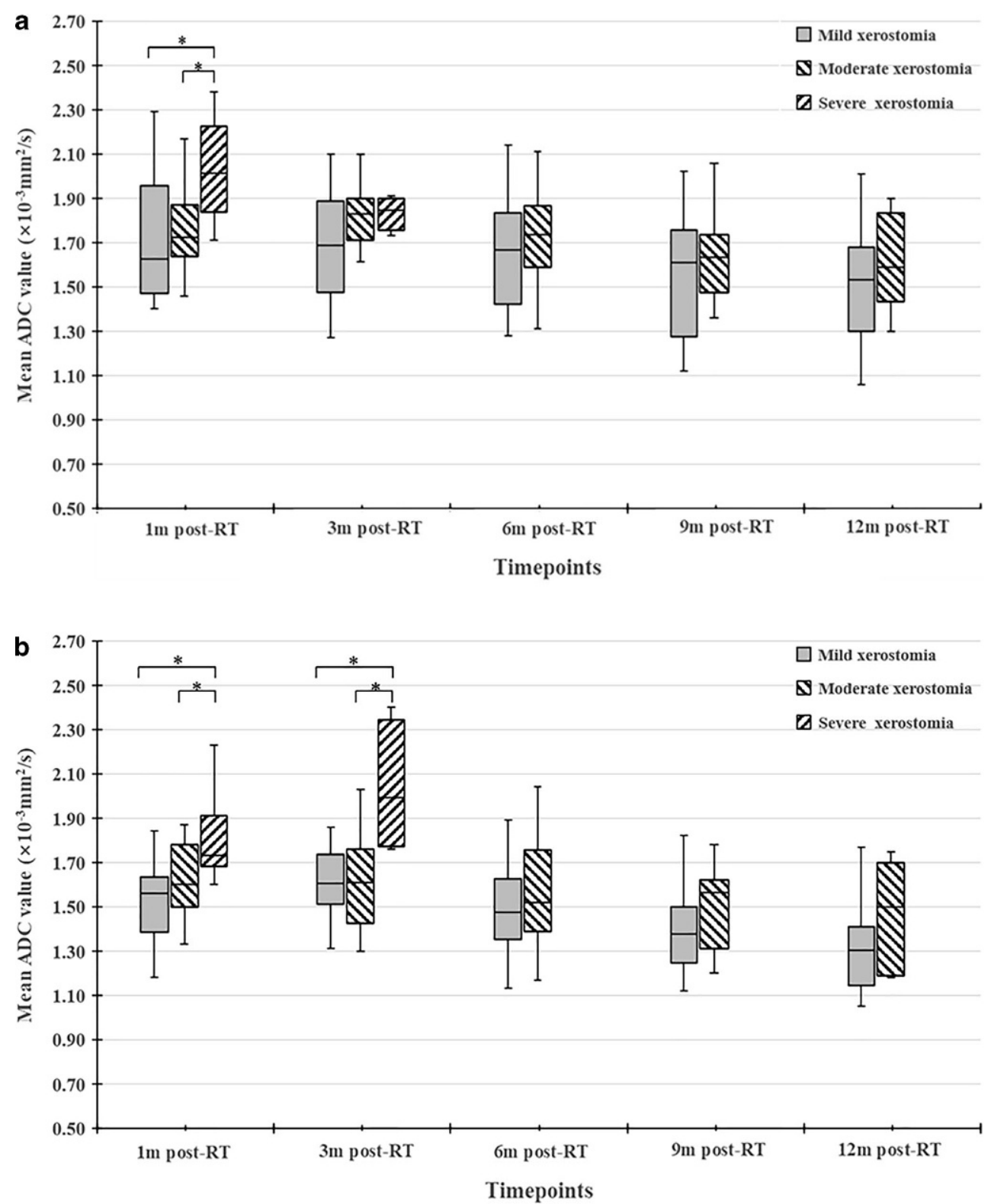

riod, ADC of both PGs and SMGs in patients with mild xerostomia and those with moderate xerostomia showed no obvious differences, while those with severe xerostomia were much lower. Meanwhile, patients with moderate subjective xerostomia presented the tendency of higher ADCs compared to those with mild xerostomia in the late post-RT period, but statistically significant differences were lacking, which might be due to the small size of our study. Based on this, we consider that although DWI is not sufficient to distinguish mild from moderate subjective xerostomia in the early post-RT period, it can fully distinguish severe subjective xerostomia and has the potential to differentiate mild from moderate subjective xerostomia in the late postRT period.
It is estimated that at least 1-year of follow-up is required to determine the effect of RT on xerostomia [32]. Thus, the follow-up period in our present study may be considered as sufficient to provide adequate information in terms of xerostomia following RT. Additionally, the inter- and intraobserver reproducibility of ADC of both PGs and SMGs was excellent, indicating high reliability of the measurement of DWI parameters of PGs as well as SMGs.

This preliminary study has some limitations. First, despite being larger than previous DWI studies on evaluation of radiation-induced salivary gland dysfunction, the sample size was still small, especially for patients with severe subjective xerostomia. Many more samples with different degrees of xerostomia should be included in future studies. Second, our study employed three continuous slices cover- 
ing the largest area of both PGs and SMGs to represent the entire salivary glands for analysis rather than including all slices. In consideration of intra-tissue heterogeneity, earlier research [33] reported regional differences in radio-sensitivity inside the salivary glands, so sampling errors could not be avoided. Regional analysis of ADCs in salivary glands should be considered in further research. Third, it has been verified that $A D C$ is not only affected by water molecular diffusion but also by microvascular perfusion [34]. The advanced intravoxel incoherent motion DWI (IVIM-DWI) technique can separate contributions of diffusion and perfusion, which makes it more informative than DWI for assessing radiation-induced salivary gland damage. Despite this, some current deficiencies such as a much longer acquisition time and a complex post-processing procedure restrict clinical applications of IVIM. Conversely, with the advantages of easily achieved fast acquisition (usually no more than $2 \mathrm{~min}$ ) and simple post-processing operation, DWI is much more convenient for patients. Thus, we consider that it is more easily achievable and suitable to use DWI as the follow-up method for evaluation of salivary gland function after RT. Last but not least, the impact of chemotherapy on salivary gland function is currently unclear and it is still an open question owing to various antineoplastic drugs, doses, and cycles. Strigari et al. [31] found that concomitant chemotherapy had no impact on xerostomia by univariate analysis in a cohort of 63 head and neck cancer patients (44 with NPC). To the best of our knowledge, there are currently no studies on the effects of chemotherapy on ADC of salivary glands present. Preliminary results of our center showed no significant differences of ADC of both PGs and SMGs pre and post induction chemotherapy. But more samples are needed to verify the results.

\section{Conclusion}

This study showed that for both PGs and SMGs, during 1 year of follow-up post RT, the dynamic change trends of ADC were negatively paralleled to those of objective measurement (SFR under unstimulated and stimulated conditions) and in line with those of patient-reported subjective degree of xerostomia (XQ scores), indicating that DWI might be a useful modality for follow-up assessing radiation-induced physiological and functional changes of major salivary glands in NPC patients. Moreover, in the late post-RT period, the dynamic change rates of ADC were negatively correlated with those of SFR, and patients with moderate subjective xerostomia presented a tendency toward higher ADCs compared to those with mild xerostomia, suggesting that follow-up DWI evaluation might be a powerful evaluation tool in this period.

\section{Compliance with ethical guidelines}

Conflict of interest W.-j. Fan, F. Teng, Y.-r. Luo, W. Yu, Q. Zhang, Y.-p. Lu, and L. Ma declare that they have no competing interests.

Ethical standards This prospective study registered with number ChiCTR1900024328 in the Chinese Clinical Trial Registry was approved by the research ethics board of the Chinese PLA General Hospital. Written informed consent was obtained from all eligible patients prior to participation.

Open Access This article is licensed under a Creative Commons Attribution 4.0 International License, which permits use, sharing, adaptation, distribution and reproduction in any medium or format, as long as you give appropriate credit to the original author(s) and the source, provide a link to the Creative Commons licence, and indicate if changes were made. The images or other third party material in this article are included in the article's Creative Commons licence, unless indicated otherwise in a credit line to the material. If material is not included in the article's Creative Commons licence and your intended use is not permitted by statutory regulation or exceeds the permitted use, you will need to obtain permission directly from the copyright holder. To view a copy of this licence, visit http://creativecommons.org/licenses/by/4. $0 /$.

\section{References}

1. Chua MLK, Wee JTS, Hui EP et al (2016) Nasopharyngeal carcinoma. Lancet 387(10022):1012-1024

2. Zhang LF, Li YH, Xie SH et al (2015) Incidence trend of nasopharyngeal carcinoma from 1987 to 2011 in Sihui County, Guangdong Province, South China: an age-period-cohort analysis. Chin J Cancer 37:15

3. Konings AWT, Coppes RP, Vissink A (2005) On the mechanism of salivary gland radiosensitivity. Int J Radiat Oncol Biol Phys 62(4):1187-1194

4. Sciubba J, Goldenberg D (2006) Oral complications of radiotherapy. Lancet Oncol 7(2):175-183

5. Jellema AP, Slotman BJ, Doornaert P et al (2007) Impact of radiation-induced xerostomia on quality of life after primary radiotherapy among patients with head and neck cancer. Int $\mathbf{J}$ Radiat Oncol Biol Phys 69(3):751-760

6. Buglione M, Cavagnini R, Di RF et al (2016) Oral toxicity management in head and neck cancer patients treated with chemotherapy and radiation: xerostomia and trismus (part 2). Literature review and consensus statement. Crit Rev Oncol Hematol 102:47-54

7. Houweling AC, Philippens MEP, Dijkema T et al (2010) A comparison of dose-response models for the parotid gland in a large group of head-and-neck cancer patients. Int $\mathrm{J}$ Radiat Oncol Biol Phys 76(4):1259-1265

8. Ma SJ, Rivers CI, Serra LM et al (2019) Long-term outcomes of interventions for radiation-induced xerostomia: a review. World J Clin Oncol 10(1):1-13

9. Kam MK, Leung SF, Zee B et al (2007) Prospective randomized study of intensity-modulated radiotherapy on salivary gland function in early-stage nasopharyngeal carcinoma patients. J Clin Oncol 25(31):4873-4879

10. Nutting CM, Morden JP, Harrington KJ et al (2011) Parotid-sparing intensity modulated versus conventional radiotherapy in head and neck cancer (PARSPORT): a phase 3 multicentre randomised controlled trial. Lancet Oncol 12(2:127-136

11. Lee TF, Liou MH, Ting HM et al (2015) Patient- and therapy-related factors associated with the incidence of xerostomia in nasopharyngeal carcinoma patients receiving parotid-sparing helical tomotherapy. Sci Rep 5:13165 
12. Eisbruch A, Rhodus N, Rosenthal D et al (2003) How should we measure and report radiotherapy-induced xerostomia? Semin Radiat Oncol 13(3):226-234

13. Tenhunen M, Collan J, Kouri M et al (2008) Scintigraphy in prediction of the salivary gland function after gland-sparing intensity modulated radiation therapy for head and neck cancer. Radiother Oncol 87(2):260-267

14. Gupta T, Hotwani C, Kannan S et al (2015) Prospective longitudinal assessment of parotid gland function using dynamic quantitative pertechnate scintigraphy and estimation of dose-response relationship of parotid-sparing radiotherapy in head-neck cancers. Radiat Oncol 10(1):371

15. Dirix P, De Keyzer F, Vandecaveye V et al (2008) Diffusionweighted magnetic resonance imaging to evaluate major salivary gland function before and after radiotherapy. Int $\mathrm{J}$ Radiat Oncol Biol Phys 71(5):1365-1371

16. Doornaert P, Dahele M, Ljumanovic R et al (2015) Use of diffusion-weighted magnetic resonance imaging (DW-MRI) to investigate the effect of chemoradiotherapy on the salivary glands. Acta Oncol 54(7:1068-1071

17. Juan CJ, Cheng CC, Chiu SC et al (2015) Temporal evolution of parotid volume and parotid apparent diffusion coefficient in Nasopharyngeal carcinoma patients treated by intensity-modulated radiotherapy investigated by magnetic resonance imaging: a pilot study. PLoS ONE 10(8):e137073

18. Loimu V, Seppälä T, Kapanen M et al (2017) Diffusion-weighted magnetic resonance imaging for evaluation of salivary gland function in head and neck cancer patients treated with intensity-modulated radiotherapy. Radiother Oncol 122(2):178-184

19. Zhang Y, Ou D, Gu Y et al (2018) Evaluation of salivary gland function using diffusion-weighted magnetic resonance imaging for follow-up of radiation-induced xerostomia. Korean J Radiol 19(4):758-766

20. Houweling AC, van den Berg CAT, Roesink JM et al (2010) Magnetic resonance imaging at 3.0T for submandibular gland sparing radiotherapy. Radiother Oncol 97(2):239-243

21. Saarilahti K, Kouri M, Collan J et al (2006) Sparing of the submandibular glands by intensity modulated radiotherapy in the treatment of head and neck cancer. Radiother Oncol 78(3):270-275

22. Du L, Zhang XX, Feng LC et al (2017) Propensity score matching analysis of a phase II study on simultaneous modulated accelerated radiation therapy using helical tomotherapy for nasopharyngeal carcinomas. Bmc Cancer 17(1):582
23. Teng F, Fan W, Luo Y et al (2019) Reducing xerostomia by comprehensive protection of salivary glands in intensity-modulated radiation therapy with helical tomotherapy technique for head-and-neck cancer patients: a prospective observational study. Biomed Res Int 2019:2401743

24. Cheng SCH, Wu VWC, Kwong DLW et al (2011) Assessment of post-radiotherapy salivary glands. Br J Radiol 84(1001):393-402

25. Nagler RM, Baum BJ, Miller G et al (1998) Long-term salivary effects of single-dose head and neck irradiation in the rat. Arch Oral Biol 43(4):297-303

26. Stephens LC, King GK, Peters LJ et al (1986) Acute and late radiation injury in rhesus monkey parotid glands. Evidence of interphase cell death. Am J Pathol 124(3):469-478

27. Marzi S, Forina C, Marucci L et al (2015) Early radiation-induced changes evaluated by intravoxel incoherent motion in the major salivary glands. J Magn Reson Imaging 41(4):974-982

28. Cox JD, Stetz J, Pajak TF (1995) Toxicity criteria of the Radiation Therapy Oncology Group (RTOG) and the European Organization for Research and Treatment of Cancer (EORTC). Int J Radiat Oncol Biol Phys 31(5):1341-1346

29. Meirovitz A, Murdoch-Kinch CA, Schipper M et al (2006) Grading xerostomia by physicians or by patients after intensity-modulated radiotherapy of head-and-neck cancer. Int J Radiat Oncol Biol Phys 66(2):445-453

30. Hawkins PG, Lee JY, Mao Y et al (2017) Sparing all salivary glands with IMRT for head and neck cancer: Longitudinal study of patientreported xerostomia and head-and-neck quality of life. Radiother Oncol 126:68-74

31. Strigari L, Benassi M, Arcangeli G et al (2010) A novel dose constraint to reduce xerostomia in head-and-neck cancer patients treated with intensity-modulated radiotherapy. Int J Radiat Oncol Biol Phys 77(1):269-276

32. Memtsa PT, Tolia M, Tzitzikas I et al (2017) Assessment of xerostomia and its impact on quality of life in head and neck cancer patients undergoing radiation therapy. Mol Clin Oncol 6(5):789-793

33. van Luijk LP, Pringle S, Deasy JO et al (2015) Sparing the region of the salivary gland containing stem cells preserves saliva production after radiotherapy for head and neck cancer. Sci Transl Med 7(305):305ra147

34. Zhou N, Chu C, Dou X et al (2016) Early evaluation of irradiated parotid glands with intravoxel incoherent motion MR imaging: correlation with dynamic contrast-enhanced MR imaging. Bmc Cancer 16(1):865 